\begin{abstract}
This chapter presents reflections on the authors experience in conducting ethnographic research in a setting in which they had an existing professional role as a registered and practicing nurse. The position held and how this was negotiated within the research and clinical environment highlights the role ethnography can play for nurses researching in healthcare settings. In particular, this chapter highlights the positional complexities of adopting both 'insider' ethnographer and professional roles in a health care context, and the insights this generated around the partial forms of knowledge that emerge. The dual status of the researcher providing a unique position in a complex setting and shaped the generation of ethnographic findings.
\end{abstract}

\title{
Biography
}

Alison F Wood, PhD, is a Lecturer at Edinburgh Napier University in the School of Health and Social Care. She is also a registered nurse with specific clinical interests in renal and haemodialysis care which was the main focus of her PhD studies at the University of Edinburgh. Since completing her PhD, research interests consider biosciences in nurse education as well as continuing to work with renal and haemodialysis patients, families and staff alongside exploring the use of creative and visual research methods. 


\section{Ethnography and Ethics in your own Workplace: Reconceptualising dialysis care from an insider nurse researcher}

\section{Introduction and Background}

This chapter reflects on my experience conducting ethnographic research in a setting in which I had an existing professional role as a registered and practicing nurse. The position I held and how this was negotiated within the research and clinical environment highlights the role ethnography can play for nurses researching in healthcare settings. In particular, this chapter highlights the positional complexities of adopting both 'insider' ethnographer and professional roles in a health care context, and the insights this generated around the partial forms of knowledge that necessarily emerge. The specific research objectives were to explore patient and nursing staff experiences of 'direct patient care', a phrase used within time efficiency programmes and time in motion studies, which are part of professional audit practices employed to classify how a nurse spends their time. My ethnographic approach utilised participant observations alongside fieldnotes, informal questioning, photographs and semi-structured interviews to understand 'direct patient care' and how it was experienced in a haemodialysis unit for those suffering from established renal failure. The unit was an outpatient department and part of a large acute hospital in Scotland, where I held a post as a registered nurse prior, during and after the research was undertaken.

Established renal failure is a condition which requires long-term treatment; one treatment option is haemodialysis which usually involves regular, three times weekly visits to a haemodialysis unit. Nurses and support workers spend time with and care for the patients when they are receiving this three-to-five hour treatment. I was 'in the field' conducting fieldwork over a nine-month period, although I had worked in the unit in a clinical role for two years prior to this, and continued to do so on 'non-research days' through the duration of my fieldwork. Moving between different perspectives in 'the unit' was made possible due to 
my status as both an insider and outsider to the field site. As a nurse and well-known staff member I was very aware of my shifting status from nurse to ethnographer.

In this chapter I use my dual status to explicate the formation of my field-sites and the ethical questions these raised. It was at the interface of my insider/outsider roles that my ethnographic findings began to emerge. I argue that my unique and blurred positions allowed access to participants and data in a complex setting and shaped the generation of my ethnographic findings around how the concept of 'direct patient care' is performed and experienced in a clinical setting.

\section{Haemodialysis}

Haemodialysis is one life-saving treatment option for people who have kidney failure. People, or patients, who have very little or no kidney function, receive haemodialysis by being connected to a machine which replicates some kidney functions and removes electrolytes and fluid from the blood. Without this treatment, or other renal replacement therapies e.g. kidney transplant, there are significant health implications and the constant concern of mortality (Thomas 2014). In Scotland, the numbers of patients who receive this in-hospital treatment is increasing, as are the numbers who suffer from kidney failure (Scottish Renal Registry 2016).

The unit where I conducted my research was part of a large acute hospital. There are 38 haemodialysis stations and these were generally all in-use three times a day. Patients attended either in the morning, afternoon or evening and over 150 patients attend and receive treatment at the unit in their 'usual' spot alongside the same patients three times a week. When patients attend for their treatment they are attached to a machine for the duration, somewhere between three to five hours, which is prescribed due to their requirements and blood results. Once attached to the machine they cannot move around. There were five 
distinct rooms where the majority of care and interactions with staff took place and nursing staff are allocated to a room where they normally remain for the day. At the unit, treatment and care is delivered by over 60 registered nurses and nine healthcare support workers.

\section{Nursing and Ethnography}

Within the field of nursing research, ethnography has been utilised to examine the views and experiences of people within a particular area, setting or aspect of health care from an 'insider's' perspective, thus generating distinct kinds of knowledge that arise only through connection with, and embeddedness in the very practices and contexts of inquiry (Thorne 1991, Roper and Shapira 2000). From Madeline Leininger's pioneering work (1970, 1978, $1985,1988)$ as a professional nurse conducting anthropologically-informed ethnographic research to explore understanding of the role of culture in nursing and in patients' experiences of health care, ethnography in nursing has gained further ground and is becoming increasingly popular (Savage 2000a, Draper 2015). Robinson (2013:19) recently claimed 'ethnography is the future of nursing science', highlighting the potential of this methodology to explore a range of perspectives that are relevant to nursing practice, foregrounding understanding of the meaning of human behaviours and valuing the patient's own voice. Furthermore, parallels have been drawn between the practice of nursing and doing ethnography, whereby value is placed on knowing through observation (of patients, and colleagues) and through reflection on practice and positions (Savage 2000b, Borbasi, Jackson et al. 2005).

Ethnographic approaches have been previously employed in similar clinical settings to the focus of this chapter. Ashwanden (2003), for example, explored the culture of 'patientcentred care' in two haemodialysis units using an ethnographic approach, and the dominance of the haemodialysis machine in the provision of haemodialysis treatment has also been examined through ethnography (Tranter, Donoghue et al. 2009). Other research has adopted 
ethnographic approaches to explore dimensions of nursing practice in this context, including the dynamics of power that shape the delivery of quality nursing care (Bennett 2010, Bennett 2011), These different examples illustrate the wide variety of foci in which ethnographic research has been employed to explore dimensions of nursing and care experiences in renal settings. Yet, there remains an absence of more methodological reflection on how ethnographic and nursing practices intersect within a clinical context with the nurse-asethnographer, and what this means for considering the processes and spaces through which 'insider' knowledge is generated about dynamics of care-giving. In this chapter I seek to examine these tensions through my ethnographic research examining direct patient care in the haemodialysis unit where I practiced as a registered nurse.

\section{Research Design}

Fieldwork was conducted for nine months (December 2013-August 2014) where I would work one day a week (11.5 hour shift) as a nurse and visit the unit up to 4-times a week in a research capacity to provide information, consent and conduct data collection. Fieldwork included observations usually lasting 5 hours, fieldnotes and informal discussions with patients and nursing staff. I also used photographs and semi-structured interviews with a selected purposive sample to include key informants from each participant group.

Photographs were taken during the observation sessions to support the research and visualise care in the setting and the spatial and material arrangement of the unit. Initially it was through this suite of methods that my ethnographic lens sought to generate a holistic picture and context of the unit. However, as I will go on to discuss, in practice constructing a holistic representation was a complex and partial process.

Ethnography in this research allowed me to experience and witness what went on in the unit. I was able to engage with research participants whilst considering the caring practices and interactions between the staff and the patients who required regular out-patient haemodialysis 
treatment. My research design enabled me to take into account three key groups of people entangled in the performance of the concept of 'direct patient care'; nurses, support workers and patients. Ethnography allowed me to capture data from their perspectives and then construct research findings through reflection on my own position as the researcher (and nurse) and my relationship with the different groups. By observing and capturing the activities, routines, interactions and procedures of the nurses, support workers and patients I sought to gain a holistic perspective of how direct patient care was delivered and experienced in this context. However, trying to gain a holistic perspective of the group was challenging due to particular NHS ethical committee constraints.

\section{Ethical constraints and positioning in time and space:}

Ethical rules and expectations, as dictated by the NHS ethics committee who approved my research, meant I could not always access and observe all areas of patient care and treatment as an ethnographer. The ethical committee had stipulated that everyone who was in a room during an observation were required to have previously consented to being observed and participate in the study. Consequently there were spaces in which patients would congregate in large numbers such as the waiting area or outside in the ambulance bay that could not be observed because I had not received consent from all patients and staff present. At times there were over 50 patients in this space at once, and combined with different staff groups coming through this area, there were a great number of people who would not have consented to participating in my research. This appeared to exclude a significant aspect of dialysis care; the interactions between patients and staff on arrival at the unit and waiting for appointments. I found it particularly frustrating at times where spaces and events became 'unobservable', for example when seven patients in a room had given consent, but one had declined to participate. This also posed problems. On one occasion a room of patients had all consented, but the staff had not. This ethical stipulation challenged my initial aim of gaining a holistic 
picture of the haemodialysis unit. By nature of my being an ethnographer in these situations, I faced exclusion from a range of spaces, practices and interactions that I would otherwise be able to access as a nurse.

Instead, I sought to structure my observations around the patient dialysis shifts and rooms, lasting approximately five hours each time, to observe the entire process from the patient's initial arrival in the room to leaving after treatment. Arranging the observational sessions in this way provided a temporal frame for understanding patient experience in interaction with staff (both nurses and healthcare support workers). This was opposed to observing shorter periods of time, focused on different aspects of dialysis care like the starting or ending of treatment, which is typically when patient-staff interaction occurs because of the staff support required. Allowing the timeframe to be the space in which the patient occupied the dialysis unit, rather than individual activities in the process, I was able to explore more closely the entire experience from the patient's perspective. This allowed observation and understanding of how the unit functioned and supported direct patient care as a process and set of relationships rather than as a series of individual actions. Being familiar with the dialysis space and arrangements within the unit and having prior knowledge of the treatment patterns and care facilitated my interpretation of these processes. Moreover, familiarity with the shift times and patterns of practice, as a nurse, enabled me to adjust my research strategies at any given time. From my professional knowledge, I could quickly establish if the area was busy and if there was pressure on staff numbers and thereby adapt my recruitment and data collection so that it did not interfere with treatment or care. As such, a distinctive aspect of my ethnographic research design was moving between both insider and more distant positions with the tempo of the dialysis shift and the rhythms of care on the unit more generally. 
The research had imposed boundaries due to the space and consenting procedures in place in line with the ethical procedures required to be followed. The main dialysis care areas were used, but due to the requirement for all present to have provide consent the smaller sections or rooms within the unit provided helpful boundaries to ensure ethical research was being conducted. These boundaries also fitted within the routine and care delivery within the area, and therefore supported the structure of the research to observe the entire care process for the patient group and staff allowing the various aspects to be witnessed and add to the conceptualisation of care and its delivery.

\section{Distance and positioning}

Unlike with traditional forms of ethnography, privileging the development of close connections with people in the field, maintaining a form of distance from the field and participants was key to my ethnographic method in that I needed to make sure I was not being 'the nurse' when I was being 'the researcher'. My professional and ethical codes meant I had to maintain particular kinds of positioning in relation to patients and colleagues, so as to not to be seen to take advantage of my professional status as a nurse when conducting ethnographic inquiry. This double identity has been a concern for other nurse researchers (Allen 2004). I used reflexive strategies to maintain awareness of my positioning and distance relative to patients and nursing colleagues, as a researcher. These strategies included open discussion with supervisors of issues challenging my position, and the use of a reflective diary which included notes taken during and following observation sessions and interviews. Yet, this conscious positioning was often quite challenging, especially when dealing with patients and staff on the clinical/non-research days. I had to ignore my research interests and carry out my job without being affected by what I was finding out as the clinical role was my priority. I was unable to talk to staff and patients to explore things they may have said about their haemodialysis experiences when they were disclosed during a clinical day. This 
challenging scenario of having two identities - professional and research - and being conscious of my positioning was also analytically productive. In furthering my own understanding of the ethical quandaries of being an insider and conducting ethnographic research in a familiar context it helped highlight the different meanings of 'distance' in research and nursing.

A benefit of the insider position - of being 'close' to both the topic and field of research was having existing knowledge of the language and jargon used in nursing and haemodialysis treatment. However, as an ethnographer, I was also forced to reflect on my interpretation and understanding of these familiar terms. For example, 'the lull' is a phrase used by various participant groups to describe the period when patients are receiving treatment and waiting for the end of their dialysis. As a nurse, I was aware of this term and used it frequently, but repositioning myself as an ethnographer, attempting to distance myself from this nursing role, I was prompted to explore this 'lull' period in more detail. It became apparent, through my ethnographic practice, that 'the lull' was more than just a period of time where little happened (from a nursing perspective); rather, this period was a key part of the care process and crucial to patients in their care experience and feeling cared for by staff.

Two staff nurses, Moira and Karen, are sitting writing notes at the desk in room 3. They are writing in the patient folders from the morning shift, all the afternoon patients are on their machines, haemodialysis has commenced, and patients are occupied with magazines, the TV or sleeping.

The importance of this period is highlighted by a Patient when questioned;

Just keeping an eye on the machine, keeping an eye on my needles, that they are still intact, and they are firm, with the tapes, we have had those problems in the past, 
especially if I fall asleep, that somebody is keeping an eye on me, and I feel quite confident that they are (Emma, Patient).

By creating a sense of distance from the expert position as a nurse, I was forced, as an ethnographer, to confront and interrogate practices, experiences and categories of meaning around dialysis care in new and different ways.

Another element of the process of positioning and distinguishing between my professional and ethnographic roles concerned my physical appearance and presentation. It was an ethical requirement to use clear behavioural or social signs to differentiate me from my nursing role and job, and also from the other nurses and staff members, when I was conducting research. This was primarily achieved by not participating as a nurse in the regular activities and keeping a distance from the usual staff and practices, but also by holding a notebook and writing fieldnotes which looks very different to the normal nursing position. Alongside this, I made efforts to look different to my regular nursing job through my choice of attire. I wore 'research scrubs' which, while still appropriate for spending time in a clinical setting, visually distinguished me from my existing professional nursing uniform and role, and from other professional staff. Moreover, my different uniform allowed me to feel different, and not quite part of my usual job or position, as well as highlighting to others that I had switched positions. This shift in appearance enabled me to access a different perspective on dialysis care in ways I would not be able to in my usual professional position.

Finally, I used my physical positioning in care situations as a mechanism to distance myself from the role of nurse, when conducting research to avoid unethical and unprofessional practice of participating in care and clinical activities while conducting ethnography. An example which nicely illustrates the different positions I took was the morning 'safety brief' where all staff would come together to hear important updates and staff allocations for the 
shift ahead. This took place at the start of every shift before any patients arrived. On research days I would not participate in this or be part of the group discussions by sitting to the side, rather than standing with the staff group. I could witness and document this event if all present had consented. In this way, I was actively distancing myself from my usual clinical role, but also demonstrating to others - my research participants - the particular role and position I was adopting on that day.

\section{Blurring of positions: crossing boundaries in space and time}

As described above, I adopted a number of different strategies within my ethnographic research to differentiate my roles and positions between research and clinical working days. However, there were occasions where maintaining distance and differentiating between researcher / nurse identities was particularly challenging. Rather than consider these as 'failures', I will discuss how these offered analytical insight into my ethnographic position and data generation which allowed the construction of an understanding of direct patient care and how it was delivered in the haemodialysis setting. Care was identified to be delivered in two ways; actively and passively, where active care focused on the engagement between the staff and patient and passive care in opposition demonstrated care was experienced through the availability and visibility of staff.

During the research, the boundary between my two roles became blurred on occasion, due to unfolding events in the clinical spaces in which I was conducting ethnography. For example, during one observation, I was required to put down my notebook and assist the nurse in the room with a situation involving a patient, captured in my field notes:

I can see that Marie-Anne needs some assistance but she cannot go anywhere as she is holding the bleeding fistula site, she is looking around but there is no staff in the room. There is no one else available in the room 
to get equipment from the trolley which is about 10 feet away so I get up to get the equipment for her. I open the packs up and put them out and also offer a suggestion of advice to use another type of dressing which I have also laid out which will help stop the bleeding 'take that off, use this and start again' I say. Marie-Anne is an experienced nurse but I want to make sure she is ok with the current situation and offer advice. I then wash my hands beside the patient and then sit back down with my notebook and continue the observation *writing down what has happened and my involvement*

Ross (patient): you're meant to be watching (directed to me)

Marie-Anne: bet that's given you something to write about (making a joke)

Ross: 'I had to oblige.

As there was no one else who was in the room to pass the nurse some equipment she required to stem the bleeding, I felt compelled to put down my notebook and pass and open the bandages and equipment she needed. From my professional role, I knew which dressings were needed from the trolley and how to provide another nurse instruction. Despite the minimal interaction from myself, the duty of care to support the patient and nurse in this situation was priority here. If I had not provided equipment my professional duty as a nurse would be have been undermined and the patient would have been at risk of harm. This situation further highlighted the importance to patients of feeling safe and cared for due to the availability and visibility of staff alongside the direct engagement with patients to provide elements of care. The relationship that nursing staff have with patients to help and support them underpins this care delivery and I was unable and unwilling to neglect this in this research situation. 
It was crucial to write into my research protocol the professional duty of practice which underpinned the conduct of my research, and which therefore shaped my positioning as a nurse and ethnographic researcher. Despite trying to maintain a distance as a researcher conducting data collection and analysis, I followed the Nursing and Midwifery Council's (NMC) code of conduct as part of my research role, as my professional code undermines the research position when patient safety is concerned. The NMC code of conduct stipulates that key to nursing is preserving patient and public safety, alongside prioritising patients and promoting professionalism and trust, and as such that in an emergency situation I would be required to step in to assist the staff in whatever way is deemed appropriate to follow the code. Therefore, it was my very 'insider' position as a nurse-researcher, and the codes and values on which my nursing role rests, that led me, on occasion, to cross the boundary between ethnographer and practitioner. This complicated my attempts to create distance between these two positions through spatial and temporal positioning strategies, and also highlights the complexity of traditional ethnographic approaches such as 'participantobservation' in clinical contexts such as this. This approach and the challenges I required to engage with assisted me in reflexive practice and exploring care and the various perspectives from the participants through this position.

In addition to these blurrings of positioning within the spaces in which clinical activity unfolded, there were also challenges to my nurse-researcher distinction due to the temporal dimension of my fieldwork. Over the course of the fieldwork, the group of patients on whom my research was (partly) focused changed. Some patients no longer required dialysis, due to either receiving a transplant, withdrawal from dialysis or dying, and new patients began receiving care and treatment. Therefore, some patients who started attending the unit did not know me as a nurse but as a researcher. Similarly some nursing staff moved on or started working on the unit during my fieldwork, and since I was part-time they often knew me more 
as a researcher than as a nurse. This identity confusion across the participants over time required continual attention from me, to explain who I was to different people and what I was doing at various different points during the fieldwork. This also required explicit acknowledgement that my (changing) position could be confusing and blurred. The shifting participant groups, both over the course of the fieldwork and on a day-to-day basis, also shaped my use of space for research whereby who had and had not consented to participate in any given space may change. My research boundaries were complex due to the constantly changing staff and patient group in the area, which were then further stratified by the distribution of consenting and non-consenting individuals.

There were concerns voiced by some staff to the researcher on research and non-research days about the kinds of information being collected, what was being observed and for what means. Although staff frequently mentioned they were pleased I was not there to pass on direct problems to the managers, some queried whether I would be 'spying' on them. On these occasions I pointed to the information sheet for guidance which stated clearly the purpose of the research. The sheet gave staff space to make a decision on participation independently and in their own time. As the research progressed, staff began to trust me as a researcher and felt less threatened by my presence and potential whistleblowing actions. Staff began to talk in front of me discussing patients, including me in conversations during their shifts and became less concerned with watching me and what I was doing as fieldwork progressed. Trusting me as a colleague, and as both a practitioner and researcher was important for supporting data collection and my ethnographic position.

Interestingly there was a complexity around participation and consent, where patients would openly identify their participation within the research and fieldwork. At points staff and patients would really demonstrate their knowledge of their participation in the research process. There were occasions where staff and patients would joke, saying things like: 'did 
you see that - do I need to do it again?' demonstrating their understanding of my observations. These moments also performed a different researcher-participant dynamic where participants were potentially doing things for the researcher's benefit.

\section{Re-entering the field post-fieldwork}

After the fieldwork was completed, although my position as ethnographer dissolved, my role as a nurse continued following the same part-time working patterns as during the fieldwork period. This presented new challenges as I remained 'in the field', ostensibly in the same capacity as nurse, but now having passed through the position of ethnographer. For example, both patients and staff who had participated and also declined consent for participation, began regular questioning about when the research was finished, what I had found out and expressed wishes to read the thesis. Even some patients who had not been receiving care during the fieldwork period showed interest in my research, despite not having any involvement in the project. This showed a need for me to continue managing the dynamic between my roles as ethnographer and nurse even beyond the formal time and space in which the ethnography was conducted.

Re-entering the field (only) as nurse also highlighted challenges to the relationships I held with colleagues. During the fieldwork period, wherein I attempted to maintain distance between my roles as nurse and researcher, I did not actively engage with staff social events to avoid feeling like I was exploiting friendships or blurring boundaries. Following the end of data collection, it took me a while to start to integrate back into the team as a colleague and participate in these sorts of situations. Whilst data analysis continued after leaving the field, staff would continue to ask about the data collected and the results of the research. This was problematic and I had to maintain a position of ethnographer and the associated ethical obligations. I could not discuss and engage in discussions about my research progress 
because of the risk of it affecting my analysis. This highlights that whilst the fieldwork had ceased, the interest and my researcher role was still present to the staff and patients.

The research focused on nursing practice and within the area there is an appetite to engage with research and evidence-based practice and to utilise research findings to improve care and experiences.

\section{Conclusions}

The chapter has illustrated the ways in which, as a nurse and ethnographer, I attempted to maintain the separation of roles which is deemed good practice in biomedical research ethics terms. However, the dynamics of the spaces and tempos through which my research 'object' - the delivery and experience of direct patient care in a dialysis unit - unfolded during the fieldwork period meant that the separation between my dual identities and position was complex and at times blurred. The 'insider' perspective I held, as a nurse practising within my field site, was valuable for navigating these dynamics to ensure my research practice did not interfere with the delivery of care, and for recognising when the distant ethnographic position should be abandoned to ensure the protection of the patient was upheld. However, as an ethnographer, I was also forced to reconsider and reflect upon my insider knowledge, adopting a different perspective and interpretation of the practices and language of caregiving and receiving on a dialysis unit. This chapter adds to increasing literature exploring the value of ethnography for nursing research (Savage 2000a, Morse 2016, Ryan 2017), and highlights the importance of acknowledging the multiple and potentially conflicting identities that are inevitable when conducting in-depth, situated enquiry in a clinical context, underpinned by professional, clinical knowledge and codes of conduct. Furthermore, this chapter extends wider debates around ethnography as a methodology in terms of how feasible a 'holistic' understanding of any field is, and the varying positions and roles the ethnographer might undertake in seeking to engage with and understand the people and practices of that 
field. As a profession based on values of reflection and observation (Savage 2000b, Borbasi, Jackson et al. 2005), as well as direct engagement with patients, nursing seems well positioned to embrace ethnography as a methodology for researching health and healthcare, and to prompt important questions for how positionality, identity and knowledge intersect through ethnography.

\section{References}

Allen, D. (2004). "Ethnomethodological insights into insider-outsider relationships in nursing ethnographies of healthcare settings." Nursing Enquiry 11(1): 14-24.

Ashwanden, C. (2003). "Discovering the Culture of Haemodialysis Units: an Ethnography." EDTNA.ERCA Journal 29(4): 192-194.

Bennett, P. (2011). "Technological intimacy in haemodialysis nursing." Nursing Inquiry 18(3): 247-252.

Bennett, P. (2010). "Satellite dialysis nursing: technology, caring and power." Journal of Advanced Nursing 67(1): 149-157.

Borbasi, S., D. Jackson and L. Wilkes (2005). "Fieldwork in nursing research: positionality, practicalities and predicaments." Journal of Advanced Nursing 51(5): 493-501.

Draper, J. (2015). "Ethnography: principles, practice and potential." Nursing Standard 29(36): 36-41.

Leininger, M. (1970). Nursing and anthropology: Two worlds to blend. New York, Wiley.

Leininger, M. (1978). Transcultural nursing concepts, theories and practices. New York, Wiley.

Leininger, M. (1985). "Transcultural care diversity and universality: A theory of nursing." Nursing and Health Care 6(4): 209-212.

Leininger, M. (1988). "Leininger's Theory of Nursing: Cultural Care Diversity and Universality." Nursing Science Quarterly 1(4):152-160.

Morse, J.M. (2016). "Underlying Ethnography." Qualitative Health Research 26(7): 875-876.

Robinson, S.G. (2013). "The Relevancy of Ethnography to Nursing Research." Nursing Science Quarterly 26(1): 14-19.

Roper, J.M. and J. Shapira (2000). Ethnography in Nursing Research. CA, Sage Publications. Savage, J. (2000a). "Ethnography and health care." British Medical Journal 321(7273): 14001402 .

Savage, J. (2000b). "Participative observation: standing in the shoes of others?" Qualitative Health Research 10(3): 324-339. 
Scottish Renal Registry (2016). Scottish Renal Registry Annual Report 2015. Edinburgh, NHS National Services Scotland. Available from:

http://www.srr.scot.nhs.uk/Publications/docs/scottish-renal-registry-report-2015-

161107.pdf?4 Accessed 20/11/17.

Ryan, G.S. (2017). "An introduction to the origins, history and principles of ethnography." Nurse Researcher 24(4): 15-21.

Thorne, S.E. (1991). "Methodological orthodoxy in qualitative nursing research: Analysis of the issues." Qualitative Health Research 1(2):178-199.

Tranter, S.A., J. Donoghue, and J. Baker (2009). "Nursing the Machine: an ethnography of a hospital haemodialysis unit." Journal of Nephrology and Renal Transplantation (JNRT) 2(3):28-41.

Thomas, N. (ed) (2014). Renal Nursing. (4th ed) Oxford, Wiley-Blackwell. 